\title{
Colchicine in Recently Hospitalized Patients with COVID-19: A Randomized Controlled Trial (COL-COVID)
}

\author{
Domingo A Pascual-Figal $\mathbb{D}^{1-3}$ \\ Aychel E Roura-Piloto ${ }^{4}$ \\ Encarnación Moral-Escudero ${ }^{4}$ \\ Enrique Bernal ${ }^{5}$ \\ Helena Albendín-Iglesias ${ }^{4}$ \\ M Teresa Pérez-Martínez' \\ Jose Antonio Noguera-Velasco ${ }^{6}$ \\ Iria Cebreiros-López ${ }^{6}$ \\ Álvaro Hernández-Vicente ${ }^{\prime}$ \\ David Vázquez-Andrés' \\ Carmen Sánchez-Pérez ${ }^{2}$ \\ Amjad Khan ${ }^{7}$ \\ Fátima Sánchez-Cabo ${ }^{3}$ \\ Elisa García-Vázquez ${ }^{4}$ \\ On behalf of the COL-COVID \\ Investigators
}

'Cardiology Department, Hospital Universitario Virgen de la Arrixaca, IMIBArrixaca, Universidad de Murcia, Murcia, Spain; ${ }^{2}$ Centro de Investigación

Biomédica en Red Enfermedades

Cardiovasculares, (CIBERCV), Madrid,

Spain; ${ }^{3}$ Centro Nacional de Investigaciones

Cardiovasculares (CNIC), Madrid, Spain;

${ }^{4}$ Infectious Diseases and Internal Medicine

Department, Hospital Universitario Virgen

de la Arrixaca, IMIB-Arrixaca, Universidad

de Murcia, Murcia, Spain; ${ }^{5}$ Infectious

Diseases and Internal Medicine

Department, Hospital Universitario Reina

Sofia, IMIB-Arrixaca, Universidad de

Murcia, Murcia, Spain; ${ }^{6}$ Clinical

Biochemistry Department, Hospital

Universitario Virgen de la Arrixaca, IMIB-

Arrixaca, Universidad de Murcia, Murcia,

Spain; ${ }^{7}$ Department of Chemistry,

University of Oxford, Oxford, UK

Correspondence: Domingo A Pascual-Figal LAIB, Despacho 2.52, Av. Buenavista s/n,

Murcia, 30120, Spain

Tel +34-868888I63

Email dpascual@um.es
Background: Colchicine has been proposed as a potential therapy in coronavirus disease 2019 (COVID-19) due to their anti-inflammatory actions.

Methods: The COL-COVID study was a prospective, randomized, controlled and openlabel clinical trial that compared colchicine added to standard treatment vs standard treatment in hospitalized COVID-19 patients that do not need mechanical ventilatory support. Colchicine was initiated within the first 48 hours of admission at a $1.5 \mathrm{mg}$ loading dose, followed by $0.5 \mathrm{mg}$ b.i.d. for one week and $0.5 \mathrm{mg}$ per day for 28 days. The study endpoints were clinical status (7-points WHO ordinal scale) and inflammatory biomarkers (IL-6 and CRP).

Results: A total of 103 patients $(51 \pm 12$ years, $52 \%$ male) were randomly allocated to colchicine arm $(n=52)$ and control arm $(n=51)$. At day 28, all patients in the colchicine group were alive and discharged, whereas in the control group, two patients died in-hospital and one patient remained hospitalized. Clinical improvement in terms of changes on WHO scale at day 14 and 28 and time to 1-point clinical improvement did not differ between the two groups. Clinical deterioration (increase of at least 1-point in WHO scale) was observed in a higher proportion of cases in colchicine group $(13.8 \%)$ vs control group $(5.8 \%)$ $(\mathrm{p}=0.303)$; after adjustment by baseline risk factors and concomitant therapies, colchicine therapy was associated with a lower risk of clinical deterioration $(p=0.030)$. Inflammatory biomarkers CRP and IL-6 concentrations course did not differ between the two arms.

Conclusion: In hospitalized COVID-19 patients, colchicine treatment neither improved the clinical status, nor the inflammatory response, over the standard treatment. Nevertheless, a preventive effect for further clinical deterioration might be possible.

Trial Registration: NCT04350320.

Keywords: colchicine, COVID-19, inflammation

\section{Plain Language Summary}

In this prospective, randomized, controlled and open-label clinical trial conducted on 103 patients, we investigated whether colchicine, added to the standard treatment in hospitalized COVID-19 patients (non-ICU), could improve the patients clinical status in terms of 7-point ordinal WHO clinical scale and inflammatory biomarkers (C-reactive protein and IL-6). After 28 days of treatment, colchicine treatment did not produce a significant advantage in terms of clinical improvement or inflammatory response over standard treatment. Nevertheless, after adjusting by baseline risk factors and therapies, colchicine therapy reduced the risk of further clinical deterioration (defined as an increase of at least 1-point in WHO scale). 


\section{Introduction}

The outbreak of COVID-19 disease caused by the novel severe acute respiratory syndrome coronavirus 2 (SARSCoV-2) has caused a worldwide emergency. COVID-19 presents a wide range of clinical forms, from asymptomatic or benign forms to severe bilateral pneumonia, multiple organ failure and death. ${ }^{1,2}$ After the initial phase of cells infection and viral replication, a significant number of patients develop an exaggerated inflammatory response, named "cytokine storm". ${ }^{3}$ This hyperinflammatory immune response is believed to result in the progression to pulmonary edema acute respiratory distress syndrome (ARDS), cardiovascular derangement, including endothelial damage and thrombosis, and progressive multiorgan failure. ${ }^{4,5}$ Amongst the implicated inflammatory axis, the interleukin-1 (IL-1) axis represents a main accelerator of such pathogenic response. ${ }^{4-6}$ In an attempt to halt this hyperinflammatory immune response, several therapies have been tested in observational and randomized clinical trials, including those specifically targeting IL-6 and IL-1 $\beta$ proinflammatory cytokines. ${ }^{7,8}$ Currently, only dexamethasone and tocilizumab have achieved scientific evidence to improve the prognosis of patients with severe COVID-19 disease. ${ }^{9,10}$

In this regard, colchicine represents a commonly used, worldwide available and cheap anti-inflammatory medication for patients with gout, other rheumatic diseases and pericarditis. ${ }^{11}$ Recently a potential role of colchicine in patients with coronary disease has also emerged. ${ }^{12}$ The anti-inflammatory actions of colchicine include the inhibition of inflammasome (NLPR3) and the IL-1 axis, as well as inhibitory effects on immune cells by impeding the polymerization of microtubules. ${ }^{13-15}$ These mechanisms are closely related to the exaggerated inflammatory response observed in severe COVID-19 patients. These features, together with its worldwide availability and a cheap agent have prompted colchicine as a potential agent for the treatment of COVID-19. ${ }^{16-18}$ In March 2020, our group as others has started clinical trials to investigate the possible role of colchicine in the prevention of COVID-19 disease progression. Deftereos et al were the first group to report the possible clinical benefit of colchicine in hospitalized COVID-19 patients. ${ }^{19}$ DellaTorre et al, wasthe first, and Tardiff et al, is the most recent group, to have reported the clinical benefits of colchicine in COVID-19 outpatients at risk of developing severe disease condition. ${ }^{20,21}$ Conversely, the RECOVERY trial has recently reported no benefit of colchicine in hospitalized patients. ${ }^{22}$ In this context, current guidelines do not recommend colchicine for the treatment of COVID-19, but encourage further research to see whether it might yet be an effective treatment.

In the present study, we present the results of a randomized, open-label and controlled clinical trial to study the benefit of colchicine added to standard treatment in hospitalized COVID-19 patients (non-ICU), (COLCOVID trial).

\section{Methods}

\section{Study Design and Population}

This is a randomized Phase III, controlled and open-label clinical trial, comparing standard of care vs standard of care plus colchicine for four weeks, in hospitalized COVID-19 patients (non-ICU) with confirmed infection of SARS-CoV-2, and within the first 48 hours of hospital admission. The study has been registered on clinicaltrials. gov with registration number NCT04350320. The studied population included patients with no severe respiratory disease at the time of recruitment; defined as the need for mechanical ventilation, non-invasive or invasive, or extracorporeal membrane oxygenation support (ECMO). The WHO 7-points scale was used to grade disease severity and clinical course as follows: 1. Not hospitalized, no limitation of activities; 2. Not hospitalized, limitation of activities; 3 . Hospitalized, not requiring supplemental oxygen; 4. Hospitalized, requiring supplemental oxygen by mask or nasal prongs; 5 . Hospitalized, non-invasive ventilation or high flow oxygen; 6 . Hospitalized, intubation and mechanical ventilation or ECMO; 7. Death. ${ }^{23}$ The inclusion criteria were: males or females above 18 years old; SARS-CoV-2 infection confirmed by reverse-transcriptase polymerase chain reaction on nasal swab (RT-PCR); admitted in hospital in the previous 48 hours with COVID-19 diagnosis and 7-points WHO clinical status of 3, 4 or 5 . The exclusion criteria were: invasive or noninvasive mechanical ventilation needed; established limitation of therapeutic effort; inflammatory bowel disease, chronic diarrhea or malabsorption; previous neuromuscular disease; any disease with an estimated vital prognosis under 1 year; severe renal insufficiency (glomerular filtration rate $<30 \mathrm{~mL} / \mathrm{min} / 1.73 \mathrm{~m} 2$ ); medical history of cirrhosis, active chronic hepatitis or severe hepatic disease defined by liver transaminases levels threefold above the normal upper limit; previous colchicine treatment for other diseases; history of allergic reaction or significant 
sensitivity to colchicine; immunosuppressive agents, including corticoids, within the previous 6 months.

Patients meeting all the inclusion criteria and none of the exclusion ones (after signing the informed consent) were centrally randomized to "colchicine" or "control" group using an automated interactive web-based system. Patients in both groups received the standard therapy for COVID-19 according to the established contemporary hospital protocols. Participants were allocated to colchicine group or control group (1:1) using the minimization method, in order to minimize the imbalance between groups for the following patient risk factors: age $(\leq 60$, $60-80$ or $\geq 80$ ), sex (male or female), time from initiation of symptoms ( $<5$ or $\geq 5$ días), cardiovascular disease (yes or no), 7-point World Health Organization (WHO) class (3 or 4) and levels of C-reactive protein $(<5,5-10$ or $>10 \mathrm{mg} /$ $\mathrm{dl})$, ferritin $(<1000$ or $\geq 1000 \mathrm{ng} / \mathrm{mL})$, D-dimer $(<500$ or $\geq 500 \mathrm{ng} / \mathrm{mL})$, IL-6 $(<70$ or $\geq 70 \mathrm{pg} / \mathrm{mL})$ and lymphocyte count $\left(<1000\right.$ or $\left.\geq 1000 / \mathrm{mm}^{3}\right)$ at randomization. Obesity was defined as body mass-index $\geq 30 \mathrm{mg} / \mathrm{m} 2$.

The study was approved by the Local Ethics Committee of Hospital Clínico Universitario Virgen de la Arrixaca and the Spanish Agency of Medications and Pharmaceutical products (AEMPS).

\section{Colchicine and Standard Therapy}

Colchicine treatment included an initial load dose of $1.5 \mathrm{mg}$ ( $1 \mathrm{mg}$ and $0.5 \mathrm{mg}$ two hours after), followed by $0.5 \mathrm{mg}$ every 12 hours during the next 7 days and $0.5 \mathrm{mg}$ every 24 hours until the completion of 28 days of total treatment. The dose was reduced by half in patients receiving ritonavir or lopinavir or with at least one of the following: reduced renal clearance $(<50 \mathrm{~mL} / \mathrm{min} /$ $1.37 \mathrm{~m} 2$ ), weight $<70 \mathrm{~kg}$ or age $>75$ years old.

Both colchicine and control group patients received the recommended standard treatment in the study. According to therapeutic recommendations, from October 2020 dexamethasone (6 mg once a day for 10 days) was considered a standard of care in patients who required supplemental oxygen (WHO scale $\geq 4$ ). Remdesivir was administered for 5 days following the Spanish Agency of Medications and Pharmaceutical Products recommendation which included: time from symptoms onset $<7$ days; two or more measurements of oxygen saturation below $94 \%$ on room air, respiratory rate $>24$ breaths/min without supplemental oxygen or Pa02/Fi02 $<300$ (Kirby index). Tocilizumab was administered at a single dose of $600 \mathrm{mg}$ and baricitinib at
$4 \mathrm{mg} /$ day for 14 days. The need for tocilizumab or baricitinib was established according to the physician on care criteria.

\section{Clinical and Laboratory Endpoints}

The main clinical endpoint was the change in the WHO 7-points ordinal clinical scale during the 28 days of treatment. In addition, the effect on IL-6 levels, as main surrogated marker of inflammatory response, was also considered as a principal endpoint; excluding those patients who received tocilizumab, as this anti-interleukin -6 receptor antibody increases IL-6 levels. Patients were followed-up every 24 hours during hospitalization, and at days 14 and 28. In addition, blood samples were collected and biobanked at admission, every 48 hours until discharge, and at days 14 and 28. The secondary endpoints included: death, ICU admission, mechanical ventilation (non-invasive and invasive) and changes in other inflammatory markers (CRP).

\section{Statistical Analysis}

The primary efficacy analysis was performed on an intention-to-treat basis. Sample size has been calculated using a significance level of 0.05 (one-sided) and $80 \%$ power for the event of an average deterioration/improvement of 1 point in favor of the intervention group, the estimated sample size was 51 patients per group (102 in total). Descriptive analyses are presented with means and standard errors for continuous variables, and frequencies and percentages for categorical variables. Differences by group were estimated with Students' $t$-test and chi-squared test, as appropriate. A multivariate logistic regression model was fit to predict worsening of at least one point in the WHO scale based on colchicine treatment, adjusting by sex, age, IL-6, ferritin, WHO scale at recruitment, corticosteroids and remdesivir. For this purpose, continuous predictors were categorized using their median values. For inflammatory markers (IL-6 and C-reactive protein) changes at 14 and 28 days were calculated. Paired Students' $t$-test was performed to detect differences by group. Patients who received tocilizumab were excluded in IL-6 analyses. All descriptive, exploratory, and statistical analyses were performed with statistical software R (v 4.0).

\section{Results}

\section{Study Population}

The study population was comprised of 103 randomized patients, 52 in the colchicine group and 51 in the control 
group (Consort flow diagram is shown in Supplemental Figure 1).

The first patient was recruited on 30th April 2020 but, due to the flattening of the hospitalizations curve, the majority of patients (75\%) were recruited from 27th October, with the last enrolled patient on 4th December 2020 (Supplemental Figure 2).

The clinical and laboratory characteristics at inclusion of both groups are shown in Table 1. No significant differences in cardiovascular risk factors or comorbidities were identified between both arms of the study. Mean age was $51 \pm 12$ years and almost half were male (52\%). The most prevalent cardiovascular risk factors were hypertension (27\%) and obesity (21\%), followed by hypercholesterolemia (24\%), and diabetes (15\%). The presence of established cardiovascular disease was low (2.9\%), and history of sleep apnea disorder was present in $6.8 \%$.

\section{Clinical Endpoints}

The 7-point ordinal scale was used for clinical assessment. Figure 1 shows the heat map of the WHO scale during the monitorization in both arms, each day during the hospitalization and at days 14 and 28 after discharge. At day 28, all patients in the colchicine arm were discharged and alive; whereas, in the control group two patients died, and one patient remained hospitalized.

The distribution of study endpoints is shown in Table 2. Neither significant differences were found in terms of WHO scale at days 14 and 28, nor time to 1-point clinical improvement. The percentage of patients who experienced clinical deterioration $(+1$ WHO scale at any time) was $13.8 \%$ in the colchicine group vs $5.8 \%$ in the control group $(\mathrm{p}=0.303)$. In terms of other secondary adverse events, the need for ICU admission $(7.8 \%$ vs $3.8 \%)$ and the progression to mechanical ventilatory support (5.9\% vs $1.9 \%)$ were also more frequent in the control group, but with no statistical significance.

We carried out multiple logistic regression analysis considering deterioration in at least 1 point in the ordinal scale as clinical endpoint. Colchicine therapy was included in the model together with other pre-defined risk characteristics (age, gender, IL-6 levels, ferritin levels) and therapies with potential benefit (dexamethasone and remdesivir). After this adjustment (Figure 2), colchicine therapy was associated with a lower risk of clinical deterioration $\quad(\mathrm{OR}=0.11, \quad \mathrm{p}=0.03)$, whereas older age $(\mathrm{OR}=82.46, \mathrm{p}=0.02)$ and higher IL-6 levels at baseline
$(\mathrm{OR}=31.36, \mathrm{p}=0.06)$ were associated with a higher risk of clinical deterioration.

\section{Safety and Concomitant Therapies}

Patients receiving colchicine have not shown any serious adverse event and no significant differences were found between arms (Supplemental Table S1). A total of nine patients received half-dose of colchicine (9\%), according to prespecified criteria and three patients (3\%) discontinued permanently colchicine due to side effects (diarrhea). Among non-serious adverse events, the most frequent was diarrhea, reaching $11.5 \%$ in colchicine group and $7.8 \%$ in the control group. The rest of reported adverse events stood below $4 \%$ in the colchicine group, and without differences compared with the control group.

Colchicine was administered with different other therapies in clinical practice as standard treatment during the study period (Supplemental Table S2). Dexamethasone was administered in most patients of the colchicine and control groups ( $81 \%$ vs $69 \%, p=0.233)$. Among antivirals, lopinavir/ritonavir were unusual (one patient in control group), whereas the use of remdesivir was common and higher in the colchicine group than the control group ( $40 \%$ vs $23 \%, \mathrm{p}=0.105$ ), as well as the use of inhaled bronchodilators (38\% vs $22 \%$, $\mathrm{p}=0.098$ ). Regarding specific therapies against cytokine storm: tocilizumab (IL-6 receptor antagonist) was used in four cases in the control group and six cases in the colchicine group ( $7.8 \%$ vs $11.5 \%, \mathrm{p}=0.764$ ); and bariticimib (Janus Kinase inhibitor) was used in none and two cases, respectively $(3.9 \%$ vs $0 \%, \mathrm{p}=0.467)$. Almost all patients received low-weight molecular heparin as per hospital standard protocol.

\section{Inflammatory Response}

We explored the effect of colchicine on IL-6, as the main inflammatory cytokine related to the inflammatory progression in COVID-19. Patients receiving tocilizumab were excluded, given that this therapy causes a raise in IL-6 levels. As shown in Figure 3, no differential effect was found between colchicine and control groups. C-reactive protein (CRP) concentrations did not differ significantly between groups either. Both IL-6 and C-reactive protein levels decreased significantly at 72 hours and thereafter up to 14 days.

\section{Discussion}

This study explored the clinical benefit of colchicine in patients who required being hospitalized due to COVID-19 
Table I Baseline Characteristics at Inclusion by Group

\begin{tabular}{|c|c|c|c|c|}
\hline & Global $(n=103)$ & Control $(n=51)$ & Colchicine $(n=52)$ & $P$ value \\
\hline Age & $51.0 \pm 12.0$ & $50.3 \pm 12.4$ & $51.8 \pm 11.7$ & 0.536 \\
\hline Male sex & $54(52.4)$ & $27(52.9)$ & $27(51.9)$ & 1.000 \\
\hline \multicolumn{5}{|l|}{ Prior history } \\
\hline Hypertension & $28(27.2)$ & $14(27.5)$ & $14(26.9)$ & 1.000 \\
\hline Dyslipidaemia & $25(24.3)$ & $14(27.5)$ & II (2I.2) & 0.606 \\
\hline Diabetes & $15(14.6)$ & $9(17.6)$ & $6(11.5)$ & 0.549 \\
\hline Obesity (BMI > $30 \mathrm{~kg} / \mathrm{m}^{2}$ ) & $22(21.4)$ & $9(17.6)$ & $13(25.0)$ & 0.503 \\
\hline Smoking & $9(8.7)$ & $3(5.9)$ & $6(11.5)$ & 0.505 \\
\hline Cardiovascular disease & $3(2.9)$ & $3(5.9)$ & $0(0.0)$ & 0.234 \\
\hline Cerebrovascular disease & $2(1.9)$ & $0(0.0)$ & $2(3.8)$ & 0.484 \\
\hline Renal insufficiency & $7(6.8)$ & $4(7.8)$ & $3(5.8)$ & 0.979 \\
\hline COPD & $\mathrm{I}(\mathrm{I} .0)$ & I (2.0) & $0(0.0)$ & 0.992 \\
\hline Obstructive sleep apnoea & $7(6.8)$ & $3(5.9)$ & $4(7.7)$ & 1.000 \\
\hline \multicolumn{5}{|l|}{ Characteristics at admission } \\
\hline Time from admission (days) & $1.3 \pm 0.6$ & $1.3 \pm 0.6$ & $1.2 \pm 0.6$ & 0.601 \\
\hline Time with symptoms (days) & $7.6 \pm 4.4$ & $8.3 \pm 5.1$ & $6.9 \pm 3.4$ & 0.107 \\
\hline WHO clinical scale & & 1.000 & & \\
\hline 3 & $34(33.0)$ & $1720(33.3)$ & $17(32.7)$ & \\
\hline 4 & $69(67.0)$ & $34(66.7)$ & $35(67.3)$ & \\
\hline NEWS scale & $2.5 \pm 1.8$ & $2.3 \pm 1.8$ & $2.7 \pm 1.9$ & 0.272 \\
\hline Systolic blood pressure $(\mathrm{mmHg})$ & $122 \pm 17$ & $123 \pm 15$ & $123 \pm 19$ & 0.918 \\
\hline Diastolic blood pressure $(\mathrm{mmHg})$ & $74.8 \pm 11.3$ & $73.7 \pm 9.8$ & $75.9 \pm 12.6$ & 0.323 \\
\hline Oxygen saturation (\%) & $96.3 \pm 1.9$ & $96.4 \pm 1.7$ & $96.1 \pm 2.2$ & 0.537 \\
\hline Temperature $\left({ }^{\circ} \mathrm{C}\right)$ & $36.5 \pm 0.6$ & $36.6 \pm 0.7$ & $36.4 \pm 0.6$ & 0.038 \\
\hline Heart rate (bpm) & $78.1 \pm 12.5$ & $77.5 \pm 12.7$ & $78.6 \pm 12.3$ & 0.655 \\
\hline Breathing rate (rpm) & $17.7 \pm 3.7$ & $17.5 \pm 3.5$ & $17.9 \pm 4.0$ & 0.614 \\
\hline Serum creatinine (mg/dL) & $0.7 \pm 0.2$ & $0.7 \pm 0.2$ & $0.8 \pm 0.2$ & 0.495 \\
\hline GFR e $\left(\mathrm{mL} / \mathrm{min} / .73 \mathrm{~m}^{2}\right)$ & $102 \pm 27.9$ & $106 \pm 31.6$ & $98.5 \pm 23.6$ & 0.162 \\
\hline Lymphocytes $\left(\times 10^{3} / \mu \mathrm{L}\right)$ & $1.2 \pm 0.5$ & $1.2 \pm 0.5$ & $1.3 \pm 0.4$ & 0.840 \\
\hline Serum D-dimer (ng/mL) & $332 \pm 297$ & $318 \pm 301$ & $345 \pm 295$ & 0.636 \\
\hline II-6 (pg/mL) & $35.1 \pm 39.1$ & $36.7 \pm 45.3$ & $33.5 \pm 32.6$ & 0.679 \\
\hline Serum ferritin $(\mathrm{ng} / \mathrm{mL})$ & $712 \pm 734$ & $756 \pm 732$ & $670 \pm 741$ & 0.557 \\
\hline AST (U/L) & $39.7 \pm 24.5$ & $39.4 \pm 25.1$ & $40.1 \pm 24.1$ & 0.888 \\
\hline C-reactive protein $(\mathrm{mg} / \mathrm{dl})$ & $7.9 \pm 6.3$ & $8.5 \pm 6.3$ & $7.3 \pm 6.4$ & 0.367 \\
\hline Troponin $\mathrm{T}(\mathrm{pg} / \mathrm{mL})$ & $7.0 \pm 11.3$ & $8.2 \pm 15.6$ & $5.9 \pm 4.5$ & 0.320 \\
\hline NT-proBNP & $\mid 39.8 \pm 290.1$ & $|4| . \mid \pm 327.8$ & $138.5 \pm 253.1$ & 0.965 \\
\hline
\end{tabular}

Note: Numbers (\%) and mean \pm standard deviation.

Abbreviations: WHO, world health organization; IL, interleukin; GFR, glomerular filtration rate (MDRD equation); NT-proBNP, N-terminal pro-B-type natriuretic peptide; ALT, alanine aminotransferase; AST, aspartate aminotransferase. 

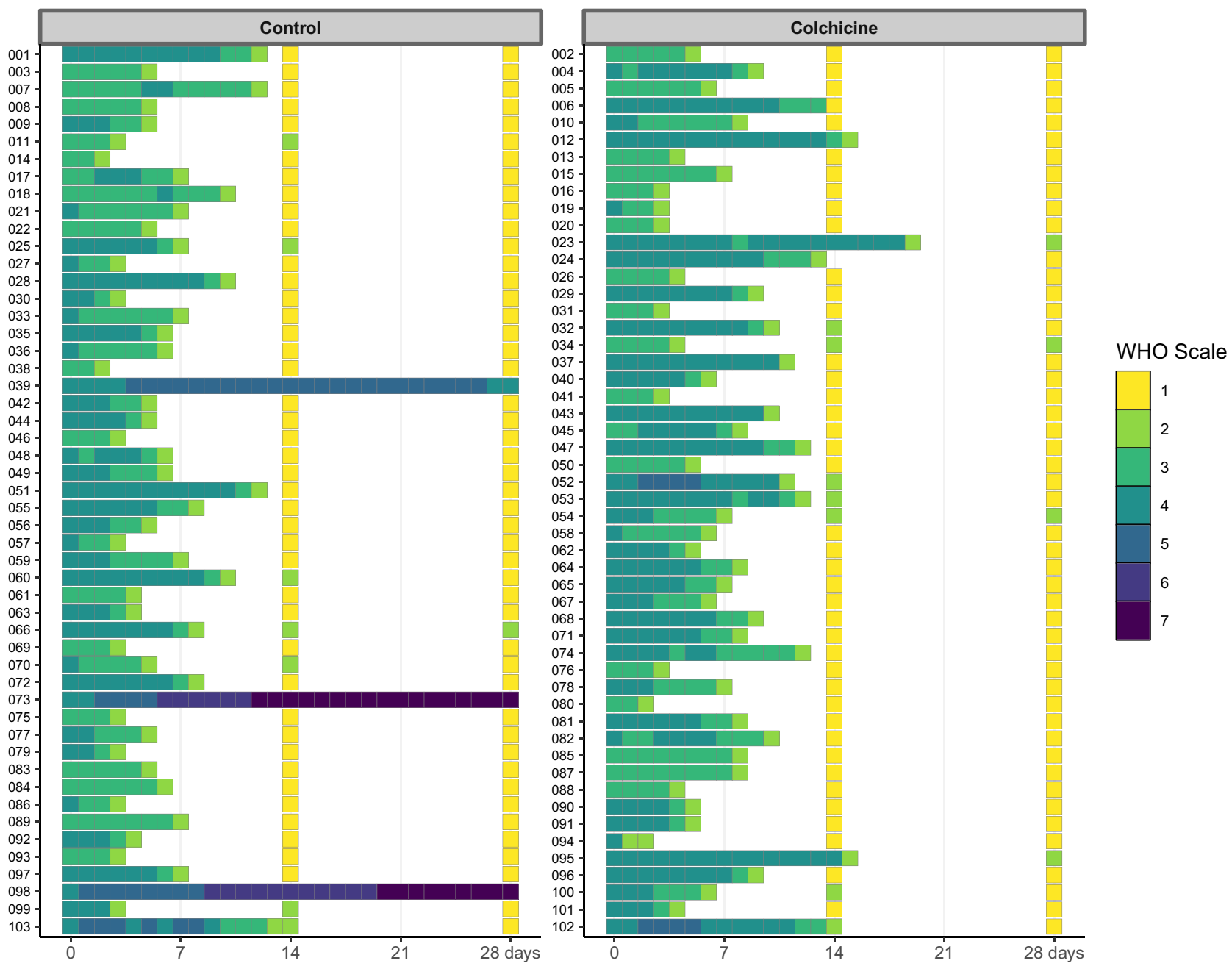

Figure I Head map showing the 7-point ordinal WHO scale across the study period of 24 weeks. Number refers to patients' identification in the study.

but did not present severity criteria yet. In this population, the administration of colchicine added to standard treatment was well tolerated and safe, but it was not associated with significant improvements in clinical condition or inflammatory markers. Only after adjustment by baseline risk factors and concomitant therapies (dexamethasone and remdesivir), colchicine was associated with a lower risk of further clinical deterioration. However, the limited sample size and the lack of effect on inflammatory parameters preclude to reach conclusions about its clinical impact and its mechanisms.

From the beginning of the SARS-CoV-2 outbreak, the presence of an exaggerated inflammatory response was identified as the main driver leading to severe complications in COVID-19 patients. $^{3-6}$ Thereby, several preexisting anti-inflammatory drugs have been proposed as candidates to prevent such response. Amongst these candidates, colchicine has been proposed based on its demonstrated anti-inflammatory actions in a range of rheumatic and cardiovascular diseases. ${ }^{13-15}$ The Greek Effects of Colchicine in COVID-19 (GRECO-19) trial was the first prospective open-label and randomized clinical trial evaluating colchicine versus usual care in hospitalized patients. ${ }^{19}$ This study of 105 patients found a significant reduction in the primary clinical outcome of a two-point deterioration on the WHO disease severity scale. In another small double-blinded randomized trial of 35 hospitalized COVID-19 patients, those patients receiving colchicine had less need for supplemental oxygen at day 7 (6\% vs 39\%) and were more likely to be discharged at day 10 (94\% vs 83\%). ${ }^{24}$ Other small control-matched studies have also described favorable clinical end-points for patients receiving colchicine added to standard care. ${ }^{25-27}$

The present study evaluated in an open-label design the effects of colchicine added to standard treatment in nonICU hospitalized COVID-19 patients. The hypothesis was that the early administration of colchicine might improve 
Table 2 Study End-Points by Group

\begin{tabular}{|c|c|c|c|c|}
\hline & Global $(n=103)$ & Control $(n=51)$ & Colchicine $(n=52)$ & $P$ value \\
\hline \multicolumn{5}{|l|}{ WHO scale } \\
\hline$\geq$ I-grade deterioration & $10(9.7)$ & $7(13.7)$ & $3(5.8)$ & 0.303 \\
\hline$\geq 2$-grade deterioration & $2(1.9)$ & $2(3.9)$ & $0(0.0)$ & 0.467 \\
\hline Days to I-grade improvement & $5.36 \pm 4.12$ & $4.96 \pm 4.71$ & $5.73 \pm 3.49$ & 0.350 \\
\hline Change at 14 days & $-2.29(1.01)$ & $-2.24(1.21)$ & $-2.35(0.76)$ & 0.579 \\
\hline Change at 28 days & $-2.48(0.96)$ & $-2.35(1.25)$ & $-2.60(0.53)$ & 0.199 \\
\hline \multicolumn{5}{|l|}{ Adverse events } \\
\hline Death & $2(1.9)$ & $2(3.9)$ & $0(0.0)$ & 0.467 \\
\hline ICU admission & $6(5.8)$ & $4(7.8)$ & $2(3.8)$ & 0.656 \\
\hline Days in ICU & $7.17 \pm 6.49$ & $8.25 \pm 8.10$ & $5.00 \pm 0.00$ & 0.621 \\
\hline Advanced respiratory support & $6(5.8)$ & $4(7.8)$ & $2(3.8)$ & 0.656 \\
\hline High outflow oxygen & $2(1.9)$ & I (2.0) & I (I.9) & \\
\hline Non-invasive ventilation & $2(1.9)$ & I (2.0) & I (I.9) & \\
\hline Invasive ventilation & $2(1.9)$ & $2(3.9)$ & $0(0.0)$ & \\
\hline Length of hospitalization & $6.18 \pm 4.40$ & $5.76 \pm 4.89$ & $6.60 \pm 3.86$ & 0.340 \\
\hline
\end{tabular}

Abbreviation: ICU, intensive care unit.

the clinical evolution in terms of "improving faster" or "impeding worsening". As previously proposed by WHO blueprint group, we used the 7-point clinical scale. The results from this study showed that colchicine did not facilitate earlier discharge or faster improvement in WHO scale. Nevertheless, colchicine administration seemed to avoid further deterioration of patients' condition when considering their previous risk factors. The small sample size population precluded an adequate statistical power to achieve significant results in the two-side comparisons. However, several findings support a potential clinical benefit for colchicine in this population. First, all patients in the colchicine group were discharged and remained alive at day 28; among patients in the control group, two patients died and one additional patient remained hospitalized at day 28. Second, in the adjusted analysis, colchicine emerged as a significant protective factor for 1-point deterioration on WHO scale. The presence of a greater benefit in specific subgroups may explain the observed protective effect after adjusting by other risk factors. Recently, the COLCORONA study published the results with a double-blinded design in COVID19 outpatients. This study showed a significant benefit in terms of lower risk of hospitalization and clinical deterioration for RT-PCR confirmed COVID-19 patients receiving colchicine. ${ }^{21}$ The impact on mortality was not significant due to the lower rate of fatalities, but was more obvious in patients with risk factors (ie, diabetes). The results of COLCORONA, GRECCO and COL-COVID point in the same direction, as they all show a trend to prevent the disease progression in a subgroup of patients. In our population, older patients and those with higher IL6 levels were at higher risk, as previously reported. ${ }^{1,2}$ However, the limited sample size precluded to identify a specific subgroup of benefit.

In the present study, we also explored the effect of colchicine on IL-6 and C-reactive protein levels, as subrogated mechanistic endpoints. However, we did not find a significant effect on these markers. No other previous data exist regarding the effect of colchicine on IL-6 concentrations. Regarding C-reactive protein, the GRECCO study did not find differences between groups, whereas Lopes et al found a greater reduction in patients receiving colchicine. ${ }^{19,24}$ We expected a positive effect of colchicine on these inflammatory markers in order to support its clinical effect. However, the fact that most patients were receiving dexamethasone at the time of inclusion might have influenced the lack of differences over the control group. Further meta-analysis would be necessary to clarify the effect on inflammatory biomarkers.

Another relevant finding of COL-COVID is the good safety profile of colchicine. No significant differences were found, and only a slightly higher rate of diarrhea was observed. These results are reassuring this drug's safety profile in hospitalized patients, with a complicated course of COVID-19 and receiving multiple drugs at the same time. Amongst the adverse events, no thrombotic events occurred. This finding supports as a spurious 


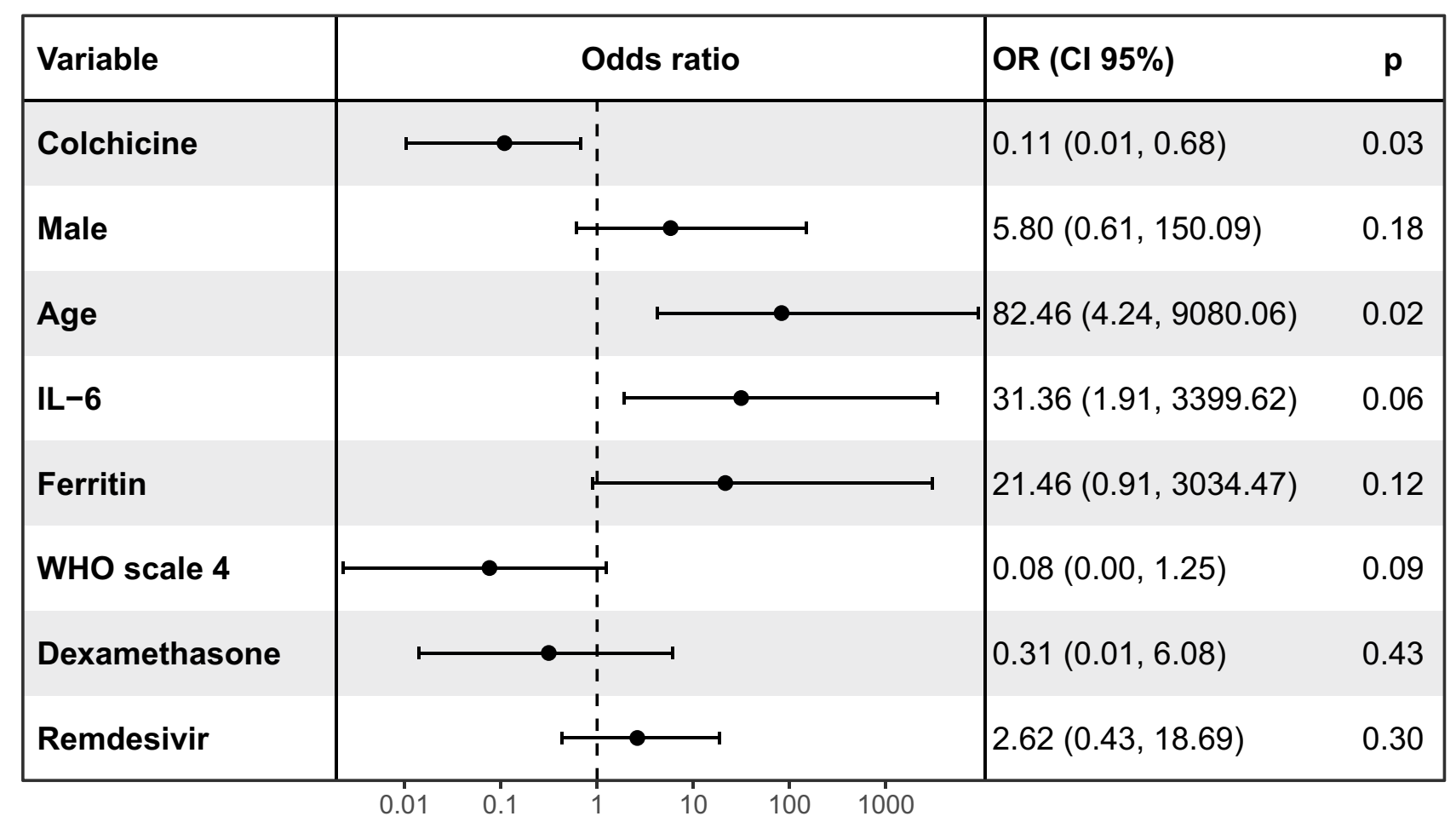

Figure 2 Adjusted multiple logistic regression analysis for the event of I-point deterioration on the WHO scale of clinical severity. Age, II-6, and ferritin were dichotomized above the median value.

A

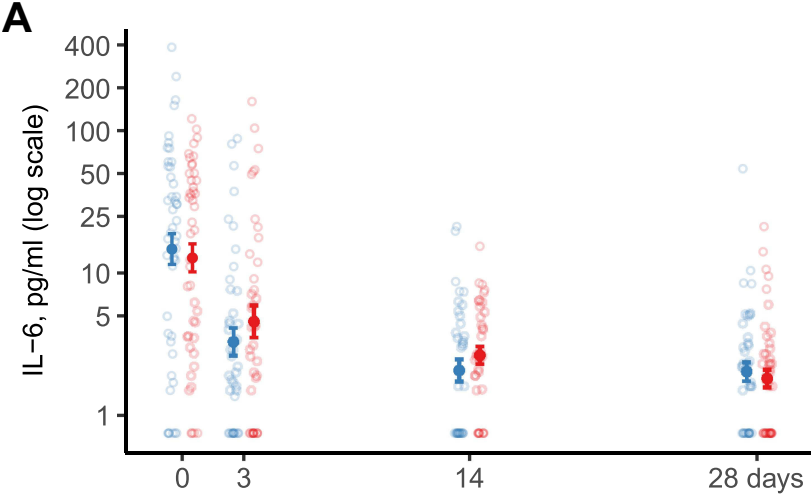

B

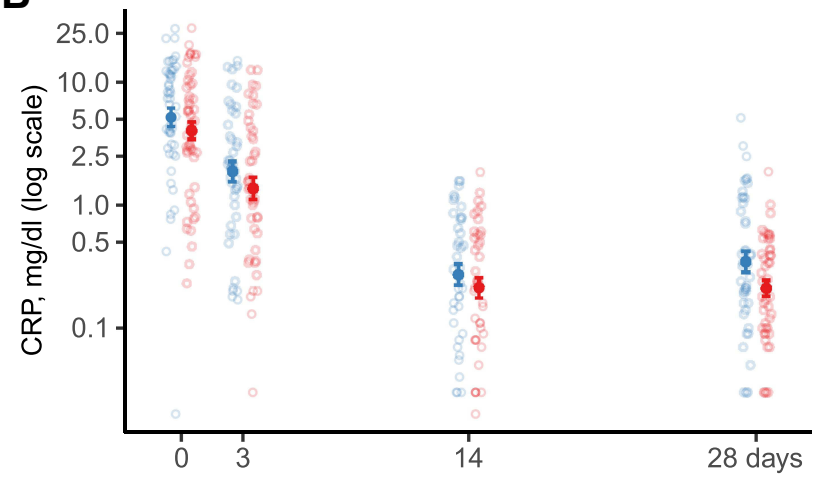

Figure 3 Concentrations of IL-6 (panel A) and C-reactive protein (panel B) in the colchicine group (red) and the control group (blue). finding the observed low rate of thrombosis in the COLCORONA trial. ${ }^{21}$

Among the limitations of our study are the small sample size and the limited number of adverse events that underpowered the ability to reach conclusions. The sample size also limited the ability to control other relevant comorbidities, such as diabetes and related therapies, ${ }^{28}$ and to investigate the effect for subgroups. Indeed, despite the controlled randomization, rates of dexamethasone, remdesivir and inhaled bronchodilators were higher in patients allocated to colchicine suggesting a higher risk from the caring physician perspective. Nevertheless, this study adds new evidence to the body of randomized clinical trials evaluating the role of colchicine in preventing the progression of COVID-19. Indeed, a recent meta-analysis showed a positive effect of colchicine in preventing the severity and mortality rate in COVID-19 patients. ${ }^{29}$ However, only three studies were randomized trials, ${ }^{20,21,24}$ which complicates reaching a conclusion regarding the effect, especially considering subgroups and populations; as the authors stated, there is a need for more randomized clinical trials. ${ }^{29}$ The RECOVERY trial included colchicine as one therapeutic candidate to compare with usual care alone in 
hospitalized patients. In the recent pre-print publication, there was no significant difference in the primary endpoint of 28-day mortality (21\% colchicine vs $21 \%$ usual care alone) ${ }^{22}$ A relevant point is that patients in the RECOVERY trial were sicker than those included in COLCORONA trial and our study. Indeed, mortality was $21 \%$ in the RECOVERY trial, $0.3 \%$ in the COLCORONA and $1.9 \%$ in our study. We may hypothesize that colchicine may have a role in preventing deterioration, as showed in COLCORONA and our study, but not in facilitating the recovery in advanced stages of the disease, as showed in the RECOVERY trial. Further research is necessary to see whether it might yet be an effective treatment targeted to prevent clinical deterioration in RT-PCR confirmed COVID-19 patients.

In conclusion, in this randomized, open-label and controlled clinical trial in hospitalized COVID-19 patients (nonICU), oral colchicine administration within the first 48 hours of hospitalization did not significantly improve the patients clinical condition,or their inflammatory markers. However, colchicine seemed to prevent further clinical deterioration after considering concomitant therapies and baseline risk variables. Further powered trials and pooled analysis are necessary to define the role of colchicine in the prevention of clinical deterioration of COVID-19 patients.

\section{Abbreviations}

SARS-CoV-2, acute respiratory syndrome coronavirus 2; COVID-19, coronavirus disease 2019; CRP, C-reactive protein; IL-6, interleukin-6; RT-PCR, real-time polymerase-chain reaction; ECMO, extracorporeal membrane oxygenation support.

\section{Data Sharing Statement}

The datasets and statistical outputs generated during the current study are available from the corresponding author on reasonable request at any time.

\section{Ethics Statement}

The study was conducted according to the guidelines of the Declaration of Helsinki and approved by the Ethics Committee of the Clinical University Hospital Virgen de la Arrixaca (EUDRACT protocol code 2020-001511-2, 14/04/2020).

\section{Acknowledgments}

We thank the Biobank Network of the Region of Murcia, BIOBANC-MUR, registered on the Registro Nacional de
Biobancos with registration number B.0000859, and the Clinical Research and Clinical Trials Unit of IMIBArrixaca. BIOBANC-MUR is supported by "Instituto de Salud Carlos III (proyecto PT20/00109) and "Instituto Murciano de Investigación Biosanitaria Virgen de la Arrixaca, IMIB".

\section{Author Contributions}

All authors made a significant contribution to the work reported, whether that is in the conception, study design, execution, acquisition of data, analysis and interpretation, or in all these areas; took part in drafting, revising or critically reviewing the article; gave final approval of the version to be published; have agreed on the journal to which the article has been submitted; and agree to be accountable for all aspects of the work.

\section{Funding}

This work has been supported by funds from 1) "Cardiology Research group" at the IMIB-Arrixaca and the University of Murcia, Murcia, Spain; 2) Centro Nacional de Investigaciones Cardiovasculares (CNIC), Madrid, Spain. Centro Nacional de Investigaciones Cardiovasculares (CNIC) is supported by the Spanish Ministry of Economy and Competitiveness (MINECO) and Pro-CNIC Foundation.

\section{Disclosure}

The authors declare no conflicts of interest in this work.

\section{References}

1. Guan W, Ni Z, Hu Y, et al. Clinical characteristics of coronavirus disease 2019 in China. $N$ Engl J Med. 2020;382(18):1708-1720. doi:10.1056/NEJMoa2002032

2. Zhou F, Yu T, Du R, et al. Clinical course and risk factors for mortality of adult inpatients with COVID-19 in Wuhan, China: a Retrospective Cohort Study. Lancet. 2020;395(10229):1054-1062. doi:10.1016/ S0140-6736(20)30566-3

3. Siddiqi HK, Mehra MR. COVID-19 illness in native and immunosuppressed states: a clinical-therapeutic staging proposal. J Hear Lung Transplant. 2020;39(5):405-407. doi:10.1016/j.healun.2020.03.012

4. Lin L, Lu L, Cao W, Li T. Hypothesis for potential pathogenesis of SARS-CoV-2 infection - a review of immune changes in patients with viral pneumonia. Emerg Microbes Infect. 2020;1-14.

5. Channappanavar R, Perlman S. Pathogenic human coronavirus infections: causes and consequences of cytokine storm and immunopathology. Semin Immunopathol. 2017;39(5):529-539. doi:10.1007/s00281-017-0629-x

6. Conti P, Ronconi G, Caraffa A, et al. Induction of pro-inflammatory cytokines (IL-1 and IL-6) and lung inflammation by COVID-19: anti-inflammatory strategies. J Biol Regul Homeost Agents. 2020;34:1.

7. Burrage DR, Koushesh S, Sofat N. Immunomodulatory drugs in the management of SARS-CoV-2. Front Immunol. 2020;11:1844. 
8. Rizk JG, Kalantar-Zadeh K, Mehra MR, Lavie CJ, Rizk Y, Forthal DN. Pharmaco-immunomodulatory therapy in COVID-19. Drugs. 2020;80(13):1267-1292. doi:10.1007/s40265-020-01367-z

9. Anon. Dexamethasone in hospitalized patients with Covid-19. N Engl J Med. 2021;384(8):693-704. doi:10.1056/NEJMoa2021436

10. Abani O, Abbas A, Abbas F, et al. Tocilizumab in patients admitted to hospital with COVID-19 (RECOVERY): a randomised, controlled, open-label, platform trial. Lancet. 2021;397:1637-1645.

11. Slobodnick A, Shah B, Krasnokutsky S, Pillinger MH. Update on colchicine, 2017. Rheumatology (Oxford). 2018;57(suppl_1):i4-i11. doi:10.1093/rheumatology/kex453

12. Aimo A, Pascual Figal DA, Bayes-Genis A, Emdin M, Georgiopoulos G. Effect of low-dose colchicine in acute and chronic coronary syndromes: a systematic review and meta-analysis. Eur $J$ Clin Invest. 2020;51(4):e13464.

13. Ravelli RBG, Gigant B, Curmi PA, et al. Insight into tubulin regulation from a complex with colchicine and a stathmin-like domain. Nature. 2004;428(6979):198-202. doi:10.1038/nature02393

14. Otani K, Watanabe T, Shimada S, et al. Colchicine prevents NSAID-induced small intestinal injury by inhibiting activation of the NLRP3 inflammasome. Sci Rep. 2016;6(1):1-10. doi:10.1038/ srep32587

15. Pope RM, Tschopp J. The role of interleukin-1 and the inflammasome in gout: implications for therapy. Arthritis Rheum. 2007;56 (10):3183-3188. doi:10.1002/art.22938

16. Yousefi H, Mashouri L, Okpechi SC, Alahari N, Alahari SK. Repurposing existing drugs for the treatment of COVID-19/SARSCoV-2 infection: a review describing drug mechanisms of action. Biochem Pharmacol. 2021;183.

17. Perricone C, Bartoloni E, Gerli R. Colchicine, an anti-rheumatic agent, as a potential compound for the treatment of COVID-19. Reumatologia. 2020;58(5):261-264. doi:10.5114/reum.2020.100088

18. Vitiello A, Ferrara F, Ferrara F. Colchicine and SARS-CoV-2: management of the hyperinflammatory state. Respir Med. 2021;178:106322. doi:10.1016/j.rmed.2021.106322

19. Deftereos SG, Giannopoulos G, Vrachatis DA, et al. Effect of colchicine vs standard care on cardiac and inflammatory biomarkers and clinical outcomes in patients hospitalized with coronavirus disease 2019: the GRECCO-19 randomized clinical trial. JAMA Netw open. 2020;3(6):e2013136. doi:10.1001/jamanetworkopen.2020.13136
20. Della-Torre E, Della-Torre F, Kusanovic M, et al. Treating COVID-19 with colchicine in community healthcare setting. Clin Immunol. 2020;217:108490.

21. Tardif J-C, Bouabdallaoui N, L'Allier PL, et al. Colchicine for community-treated patients with COVID-19 (COLCORONA): a Phase 3, randomised, double-blinded, adaptive, placebo-controlled, multicentre trial. Lancet Respir Med. 2021;19:1-9.

22. Landray M. Colchicine in patients admitted to hospital with COVID-19 (RECOVERY): a randomised, controlled, open-label, platform trial. medRxiv. 2021;2021.05.18.21257267.

23. World Health Organization. R\&D blueprint and COVID-19. World Health Organization; 2020. Available from: https://www.who.int/ teams/blueprint/covid-19. Accessed February 19, 2021.

24. Lopes MI, Bonjorno LP, Giannini MC, et al. Beneficial effects of colchicine for moderate to severe COVID-19: an interim analysis of a randomized, double-blinded, placebo controlled clinical trial. medRxiv. 2020;2020.08.06.20169573.

25. Scarsi M, Piantoni S, Colombo E, et al. Association between treatment with colchicine and improved survival in a single-centre cohort of adult hospitalised patients with COVID-19 pneumonia and acute respiratory distress syndrome. Ann Rheum Dis. 2020;79 (10):1286-1289. doi:10.1136/annrheumdis-2020-217712

26. Sandhu T, Tieng A, Chilimuri S, Franchin G. A case control study to evaluate the impact of colchicine on patients admitted to the hospital with moderate to severe covid-19 infection. Can J Infect Dis Med Microbiol. 2020;2020.

27. Brunetti L, Diawara O, Tsai A, et al. Colchicine to weather the cytokine storm in hospitalized patients with COVID-19. J Clin Med. 2020;9(9):2961. doi:10.3390/jcm9092961

28. Ivan T, Kurniawan A. Metformin use is associated with reduced mortality rate from coronavirus disease 2019 (COVID-19) infection. Obes Med. 2020;19:100290. doi:10.1016/j.obmed.2020.100290

29. Ivan T, Devina H, Halim A, Jodhinata C, Audi T, Andree Y. Colchicine treatment can improve outcomes of coronavirus 19): a systematic review and analysis. Clin Exp Pharmacol Physiol. 2021;6:823-830.
International Journal of General Medicine

\section{Publish your work in this journal}

The International Journal of General Medicine is an international, peer-reviewed open-access journal that focuses on general and internal medicine, pathogenesis, epidemiology, diagnosis, monitoring and treatment protocols. The journal is characterized by the rapid reporting of reviews, original research and clinical studies across all disease areas. The manuscript management system is completely online and includes a very quick and fair peer-review system, which is all easy to use. Visit http://www.dovepress.com/ testimonials.php to read real quotes from published authors. 